\title{
Experimental and pan-cancer genome analyses reveal widespread contribution of acrylamide exposure to carcinogenesis in humans
}

\author{
Maria Zhivagui, ${ }^{1}$ Alvin W.T. Ng, ${ }^{2,3,4}$ Maude Ardin, ${ }^{1}$ Mona I. Churchwell, ${ }^{5}$ \\ Manuraj Pandey, ${ }^{1}$ Claire Renard, ${ }^{1}$ Stephanie Villar, ${ }^{1}$ Vincent Cahais, ${ }^{6}$ Alexis Robitaille, ${ }^{7}$ \\ Liacine Bouaoun, ${ }^{8}$ Adriana Heguy, ${ }^{9}$ Kathryn Z. Guyton, ${ }^{10}$ Martha R. Stampfer, ${ }^{11}$ \\ James McKay, ${ }^{12}$ Monica Hollstein, ${ }^{1,13,14}$ Magali Olivier, ${ }^{1}$ Steven G. Rozen, ${ }^{2,3,4}$ \\ Frederick A. Beland, ${ }^{5}$ Michael Korenjak, ${ }^{1}$ and Jiri Zavadil ${ }^{1}$
}

${ }^{1}$ Molecular Mechanisms and Biomarkers Group, International Agency for Research on Cancer, Lyon 69008, France; ${ }^{2}$ Centre for Computational Biology, Duke-NUS Medical School, Singapore 169857, Singapore; ${ }^{3}$ Program in Cancer and Stem Cell Biology, Duke-NUS Medical School, 169857, Singapore; ${ }^{4}$ NUS Graduate School for Integrative Sciences and Engineering, Singapore 117456, Singapore; ${ }^{5}$ Division of Biochemical Toxicology, National Center for Toxicological Research, Jefferson, Arkansas 72079, USA; ${ }^{6}$ Epigenetics Group, International Agency for Research on Cancer, Lyon 69008, France; ${ }^{7}$ Infections and Cancer Biology Group, International Agency for Research on Cancer, Lyon 69008, France; ${ }^{8}$ Environment and Radiation Section, International Agency for Research on Cancer, Lyon 69008, France; ${ }^{9}$ Department of Pathology and Genome Technology Center, New York University, Langone Medical Center, New York, New York 10016, USA; ${ }^{10}$ IARC Monographs Group, International Agency for Research on Cancer, Lyon 69008, France; ${ }^{11}$ Biological Systems and Engineering Division, Lawrence Berkeley National Laboratory, Berkeley, California 94720, USA; ${ }^{12}$ Genetic Cancer Susceptibility Group, International Agency for Research on Cancer, Lyon 69008, France; ${ }^{13}$ Deutsches Krebsforschungszentrum, 69120 Heidelberg, Germany; ${ }^{14}$ Faculty of Medicine and Health, University of Leeds, LIGHT Laboratories, Leeds LS2 9JT, United Kingdom

\begin{abstract}
Humans are frequently exposed to acrylamide, a probable human carcinogen found in commonplace sources such as most heated starchy foods or tobacco smoke. Prior evidence has shown that acrylamide causes cancer in rodents, yet epidemiological studies conducted to date are limited and, thus far, have yielded inconclusive data on association of human cancers with acrylamide exposure. In this study, we experimentally identify a novel and unique mutational signature imprinted by acrylamide through the effects of its reactive metabolite glycidamide. We next show that the glycidamide mutational signature is found in a full one-third of approximately 1600 tumor genomes corresponding to 19 human tumor types from 14 organs. The highest enrichment of the glycidamide signature was observed in the cancers of the lung $(88 \%$ of the interrogated tumors), liver (73\%), kidney (>70\%), bile duct (57\%), cervix (50\%), and, to a lesser extent, additional cancer types. Overall, our study reveals an unexpectedly extensive contribution of acrylamide-associated mutagenesis to human cancers.
\end{abstract}

[Supplemental material is available for this article.]

Cancer can be caused by lifestyle factors, environmental or occupational exposures involving chemicals, their complex mixtures, and physical and biological agents. Many human carcinogens show shared key characteristics (Smith et al. 2016), and different carcinogens may have a spectrum of such characteristics and operate through distinct mechanisms to produce genetic alterations. Recognizable somatic alteration patterns characterize carcinogens that are mutagenic. Single-base substitution (SBS) mutational signatures can be expressed in simple mathematical terms that enable them to be extracted from thousands of cancer genomes (Alexandrov et al. 2013a, 2018). Several of the identified mutational signatures have been attributed to specific external exposures or endogenous factors through epidemiological and/or experimental studies (Alexandrov et al. 2018). The majority of the signatures re-

Corresponding authors: zavadilj@iarc.fr; korenjakm@iarc.fr Article published online before print. Article, supplemental material, and publication date are at http://www.genome.org/cgi/doi/10.1101/gr.242453.118. Freely available online through the Genome Research Open Access option. main of unknown origin, and additional, yet unrecognized, signatures are likely to be extracted from rapidly accumulating cancer genome data. Well-controlled experimental exposure systems can help identify the causes of the orphan mutational signatures and define new carcinogen-generated patterns (for review, see Hollstein et al. 2017; Zhivagui et al. 2017).

Various diet-related and iatrogenic exposures contribute to human cancer burden, involving, for instance, food contaminants (aflatoxin B1 [AFB1]) or alternative medicines (aristolochic acid [AA]) with well-documented mutagenic properties; AFB1 induces predominantly C:G>A:T and AA generates T:A>A:T transversions. These characteristic mutations, arising in preferred sequence contexts, allowed unequivocal association of exposure to AFB1 or AA with specific subtypes of hepatobiliary or urological cancers (Poon et al. 2013; Meier et al. 2014; Scelo et al. 2014; Jelaković

(C) 2019 Zhivagui et al. This article, published in Genome Research, is available under a Creative Commons License (Attribution-NonCommercial 4.0 International), as described at http://creativecommons.org/licenses/by-nc/4.0/. 
et al. 2015; Hoang et al. 2016; Chawanthayatham et al. 2017; Huang et al. 2017; Ng et al. 2017; Zhang et al. 2017).

Among dietary compounds with carcinogenic potential, acrylamide (ACR) is of interest because of its ubiquitous presence. Important sources of exposure to ACR include tobacco smoke (Mojska et al. 2016), coffee (Takatsuki et al. 2003), and a spectrum of occupational settings (IARC 1994). ACR forms in carbohydraterich foods (e.g., potatoes and cereals) heated at high temperatures, because of Maillard reactions involving reducing sugars and the amino acid asparagine (Tareke et al. 2002). There is sufficient evidence that ACR is carcinogenic in rodents (Beland et al. 2013, 2015), and it was classified by the International Agency for Research on Cancer (IARC) as a probable carcinogen (Group 2A) (IARC 1994). The associations of dietary ACR exposure with renal, endometrial, and ovarian cancers have been explored in epidemiological studies (Hogervorst et al. 2008; Virk-Baker et al. 2014; Pelucchi et al. 2015). However, accurate ACR exposure assessment by questionnaires has been difficult, whereas more direct measures of molecular markers, such as hemoglobin adduct levels, may not yield conclusive findings on past exposures (Olesen et al. 2008; Wilson et al. 2009; Xie et al. 2013; Obón-Santacana et al. 2016a, $\mathrm{b}, \mathrm{c})$. Thus, innovative well-controlled exposure model systems can improve our understanding of the ACR exposure-associated effects and risk.

Oxidation of ACR by cytochrome $\mathrm{P} 450$ produces the highly reactive electrophilic epoxide glycidamide (GA) (Segerbäck et al. 1995; Sumner et al. 1999; Ghanayem et al.2005). The Hras mutation loads in neoplasms of mice exposed to ACR or GA were higher upon exposure to GA (Von Tungeln et al. 2012), and more mutations in the $c I I$ reporter gene of Big Blue mouse embryonic fibroblasts were obtained by GA treatment in comparison to ACR (Besaratinia and Pfeifer 2003, 2004). In vivo and in vitro reporter gene mutagenesis studies showed an increased association of ACR and GA exposure with $\mathrm{T}: \mathrm{A}>\mathrm{C}: \mathrm{G}$ transitions and $\mathrm{T}: \mathrm{A}>\mathrm{A}: \mathrm{T}$ and $\mathrm{C}: \mathrm{G}>\mathrm{G}: \mathrm{C}$ transversions (Besaratinia and Pfeifer 2003, 2004; Von Tungeln et al. 2009, 2012; Ishii et al. 2015; Manjanatha et al. 2015). In addition, GA exposure induces C:G>A:T transversions (Besaratinia and Pfeifer 2004). However, these ACR- and GA-specific patterns were based on limited mutation counts and do not allow translating adequately the reported mutation types into genome-wide patterns.

Massively parallel sequencing allows studying a large number of mutations in a single sample, thus significantly enhancing the power of mutation analysis in experimental models. Analogously to human cancer genome projects, genome-scale mutational signatures can be extracted from highly controlled carcinogen exposure experiments using mammalian cells and animal models, in combination with advanced computational methods (Olivier et al. 2014; Nik-Zainal et al. 2015; Huang et al. 2017). By integrating massively parallel sequencing and DNA adduct analysis in a mammalian cell clonal expansion model (Olivier et al. 2014; Nik-Zainal et al. 2015; Huskova et al. 2017) and by computational interrogation of the Pan-Cancer Analysis of Whole Genomes (PCAWG) data, we aimed to systematically investigate the mutational signatures of ACR and GA and to determine the contribution of ACR/GA to human carcinogenesis.

\section{Results}

\section{Human TP53 mutations generated by ACR or GA treatment}

Primary Hupki MEF cultures from three different embryos (Prim_1, Prim_2, and Prim_3) exposed to ACR or GA at the predetermined cytotoxic and genotoxic conditions yielded multiple immortalized clones (Methods) (Supplemental Fig. S1) suitable for massively parallel sequencing (Olivier et al. 2014). Sanger sequencing of TP53 in the clones derived from ACR exposure (ACR clones) and GA exposure (GA clones) and spontaneous immortalization (Spont), showed that ACR clones obtained from the Prim_2 MEFs showed loss of heterozygosity in the TP53 codon 72 involving a loss of the proline allele (ACR_1 clone), and also loss of the arginine allele resulting in a hemizygous ACR_2 clone (Table 1). No TP53 mutations were observed in the Spont clones. The detection of TP53 mutations in three out of seven ACR clones and in one out of five GA clones (Table 1) provided a sound rationale for extended sequencing at the exome scale.

\section{Analysis of mutation spectra}

Whole-exome sequencing (WES) of all Spont as well as exposed clones revealed that the total number of acquired SBS did not differ markedly between the ACR and Spont clones. The Spont clones harbored on average $190($ median $=151$, range $=141-277)$ SBSs, whereas the ACR clones had on average 208 (median $=173$, range $=151-262$ ) SBSs. In contrast, the total number of SBSs was considerably increased in the GA clones, with an average of 485 SBSs (median $=448$, range $=370-592$ ) (Supplemental Tables S1, S2). This finding reveals stronger mutagenic properties of GA in the MEFs.

Principal component analysis (PCA) performed on the resulting SBS spectra unambiguously separated the GA clones from other experimental conditions (Fig. 1A). The ACR-exposed samples showed a diffuse pattern across the six SBS classes, whereas the Spont clones showed an enrichment of C:G>G:C SBS in the 5'-GCC-3' context, also present across the exposed cultures (Supplemental Fig. S2). This background mutation type appears related to the culture conditions used for the MEF immortalization assay, and its consistent formation has been observed previously (Olivier et al. 2014; Nik-Zainal et al. 2015). No significant transcription strand bias (TSB) was observed for any mutation class in the Spont or ACR clones (Supplemental Fig. S3). In the clones derived from the GA-treated primary MEF cultures, we observed an enrichment of T:A > A:T and C:G > A:T transversions and T:A> C:G transitions (Supplemental Fig. S2B), marked by significant TSB (Supplemental Fig. S3). The GA-associated clones showed lower numbers ( 25 per clone) of small insertions/deletions (indels) in comparison to the ACR (44 per clone) or Spont clones (39 per clone) (see Supplemental Tables S1, S3). Thus, higher SBS counts owing to GA treatment may selectively promote the senescence bypass and the selection, with a decreased functional contribution of indels, whereas an inverse scenario is plausible for the Spont and ACR clones, consistent with a previous report based on the Big Blue mouse embryonic fibroblasts and cII transgene (Besaratinia and Pfeifer 2005).

Variant allele frequency (VAF) analysis performed for GA clones detected a large proportion of acquired mutations manifesting at VAF between 25\% and 75\% (Supplemental Fig. S4C). Upon grouping of substitutions into bins of high (67\%-100\%), medium (34\%-66\%), and low (0\%-33\%) VAF, the predominant GA-specific mutation types (T:A>A:T, T:A > C:G, and C:G > A:T) started manifesting at high VAF and became increasingly enriched in the medium and low VAF intervals. The background $5^{\prime}-\mathrm{N}[\mathrm{T}>\mathrm{G}] \mathrm{T}-3^{\prime}$ SBS, corresponding to COSMIC signature 17 arising in cultured mouse cells including MEFs (Behjati et al. 2014; Nik-Zainal et al. 2015; Milholland et al. 2017), displayed minor, although not statistically

\section{Genome Research}

www.genome.org 
Table 1. Summary of cell lines, treatment conditions, and TP53 mutation status

\begin{tabular}{|c|c|c|c|c|c|c|c|c|}
\hline $\begin{array}{l}\text { Sample } \\
\text { ID }\end{array}$ & Embryo & Exposure & $\begin{array}{l}\text { Conc. } \\
\text { (mM) }\end{array}$ & $\begin{array}{l}\text { Exposure } \\
\text { duration } \\
\text { (h) }\end{array}$ & $\begin{array}{l}\text { Coding DNA } \\
\text { change }^{\mathrm{a}}\end{array}$ & Genomic DNA change $^{b}$ & aa change & $\begin{array}{c}\text { Codon 72 } \\
(\text { rs1042522) }\end{array}$ \\
\hline Prim_1 & E210 & - & - & - & & & & Pro/Pro \\
\hline Prim_2 & E213 & - & - & - & & & & Arg/Pro \\
\hline Prim_3 & E214 & - & - & - & & & & Pro/Pro \\
\hline Spont_1 & E213 & - & - & - & & & & Arg/Pro \\
\hline Spont_2 & E214 & - & - & - & & & & Pro/Pro \\
\hline Spont_3 & E214 & - & - & - & & & & Pro/Pro \\
\hline ACR_S9_1 & E213 & ACR & 5 & 24 & & & & Arg/Pro \\
\hline ACR_S9_2 & E213 & ACR & 5 & 24 & & & & Arg/Pro \\
\hline ACR_1 & E213 & ACR & 10 & 24 & c.881 delA & g.7577057delT & p.E294fs & Arg/- \\
\hline ACR_2 & E213 & ACR & 10 & 24 & $c .818 \mathrm{G}>\mathrm{T}$ & g. $7577120 \mathrm{C}>\mathrm{A}$ & p.R273L & Pro/- \\
\hline ACR_3 & E214 & ACR & 10 & 24 & c. $740 \mathrm{~A}>\mathrm{T} ; \mathrm{c} .839 \mathrm{G}>\mathrm{C}$ & g.7577541T $>A ; g .7577099 C>C$ & p.N247I; p.R280T & Pro/Pro \\
\hline ACR_4 & E214 & ACR & 10 & 24 & & & & Pro/Pro \\
\hline ACR_5 & E214 & ACR & 10 & 24 & & & & Pro/Pro \\
\hline GA_ $\overline{1}$ & E210 & GA & 3 & 24 & & & & Pro/Pro \\
\hline GA_2 & E210 & GA & 3 & 24 & & & & Pro/Pro \\
\hline GA_3 & E210 & GA & 3 & 24 & c. 309-310CC >TA & g.7579377-7579378GG $>$ TA & [p.Y103Y; p.Q104K] & Pro/Pro \\
\hline GA_4 & E214 & GA & 3 & 24 & & & & Pro/Pro \\
\hline GA_5 & E214 & GA & 3 & 24 & & & & Pro/Pro \\
\hline
\end{tabular}

(TP53) human TP53 gene; (Prim) primary cells; (Spont) spontaneously immortalized clones; (ACR) acrylamide-exposure derived clones; (GA) glycidamide-exposure derived clones. Each exposure condition was carried out in two biological replicates (embryos). (S9) human S9 fraction; (Pro) proline; (Arg) arginine; (Arg/-) or (Pro/-) loss of allele; (fs) frameshift; (aa) amino acid.

aNM_000546.4 coding sequence.

bhg19 genomic coordinates.

'Human polymorphic site (rs1042522).

significant, lower-VAF enrichment $\left(P=0.25\right.$, assessed by $\chi^{2}$ test $)$ (Supplemental Fig. S5). These observations suggest early effects of the GA exposure, reproducible contribution of the induced mutations to senescence bypass, and their clonal propagation during the immortalization stage.

\section{Mutational signature of GA}

Three distinct mutational signatures were extracted from all MEF clones, termed signatures A, B, and C. Signatures A and C were enriched in the Spont and ACR clones, whereas the more robust signature B was selectively enriched in the GA clones (Fig. 1B; Supplemental Fig. S6). The TSB analysis in the GA clones revealed significant enrichment of the prominent mutation types $C: G>A: T$, $\mathrm{T}: \mathrm{A}>\mathrm{A}: \mathrm{T}$, and $\mathrm{T}: \mathrm{A}>\mathrm{C}: \mathrm{G}$ (using the pyrimidine-based mutation class convention) on the transcribed strand $\left(P<0.05, \chi^{2}\right.$ test), consistent with the less efficient transcription-coupled nucleotide excision repair because of adduct formation on purines (Fig. 1C; Supplemental Fig. S3). In signature $C$ and to a lesser extent in signatures $\mathrm{A}$ and $\mathrm{B}$, we observed an admixture of a pattern identical to the COSMIC signature 17 (T:A> G:C in the $5^{\prime}-\mathrm{NTT}-3^{\prime}$ trinucleotide context), present in human cancers (notably esophageal and gastric adenocarcinomas) but also seen in AFB1-driven mouse liver cancers (Huang et al. 2017), in murine small cell lung carcinoma initiated by loss of Trp53 and Rb1 (McFadden et al. 2014), and in primary MEF-derived clones (Olivier et al. 2014; Nik-Zainal et al. 2015). This signature has been linked to cell culture conditions (Behjati et al. 2014; Milholland et al. 2017) and may be linked to oxidative stress effects on the free dGTP pool (Tomkova et al. 2018). To further refine the putative GA mutational signature from signature $\mathrm{B}$, we used extended-input nonnegative matrix factorization (NMF) by combining the MEF clone data with signature 17-rich esophageal adenocarcinoma data from the International Cancer Genome Consortium (ICGC) ESAD-UK study (Secrier et al. 2016), as well as with The Cancer Genome Atlas (TCGA) esophageal ade- nocarcinoma (ESCA) and gastric carcinoma (STAD) samples enriched for or lacking signature 17 (see Methods) (Supplemental Methods; Supplemental Figs. S6, S7). This considerably reduced (average $=47 \%$, median $=48 \%$ ) the signature 17 -specific $T>G$ peaks in signature B associated with GA treatment and resulted in a cleaner pattern (Fig. 1D; Supplemental Fig. S6). The refined GA signature retains the strand-biased enrichment of the $\mathrm{T}: \mathrm{A}>\mathrm{A}: \mathrm{T}$ transversions and $\mathrm{T}: \mathrm{A}>\mathrm{C}: \mathrm{G}$ transitions in the $5^{\prime}-\mathrm{CTG}-3^{\prime}$ and $5^{\prime}$-CTT-3' trinucleotide contexts, as well as the C:G $>\overline{A: T}$ component (Fig. 1D; Supplemental Fig. S8A; Supplemental Table S4).

\section{Quantitative DNA adduct analysis supports the GA mutational signature}

Following metabolic activation, ACR induces GA-DNA adducts at the N7 and N3 positions of guanine and adenine, respectively. Analysis using liquid chromatography-tandem mass spectrometry (LC-MS/MS) revealed the absence of these adducts in the untreated samples, as well as in MEFs exposed to ACR in the absence of S9 fraction (with levels below the limit of detection [LOD]). This suggests a lack of Cyp2e1 activity normally required for the metabolism of ACR to GA in the MEFs. Upon addition of human S9 fraction, N7-(2-carbamoyl-2-hydroxyethyl)-guanine (N7-GAGua) levels increased to 11 adducts $/ 10^{8}$ nucleotides (twice the LOD levels), suggesting limited metabolic activation of ACR despite the enzymatic activity of the $S 9$ fraction (Fig. 1E,F). In contrast, cells exposed to GA showed high DNA adduct levels, with N7-GA-Gua and N3-(2-carbamoyl-2-hydroxyethyl)-adenine (N3GA-Ade) observed at 49,000 adducts $/ 10^{8}$ nucleotides and 350 adducts $/ 10^{8}$ nucleotides, respectively, after subtracting the trace amount of contamination from the internal standard (Fig. 1E,F). These observed DNA adducts provide a possible mechanistic basis for the mutation types, the TSB, and the mutational signature arising upon treatment with GA, the reactive metabolite of ACR. 
A

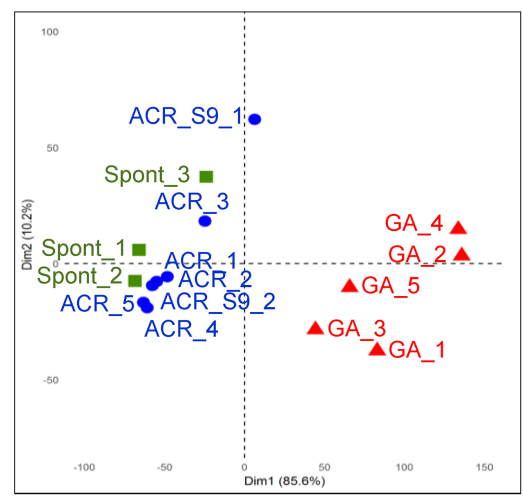

C

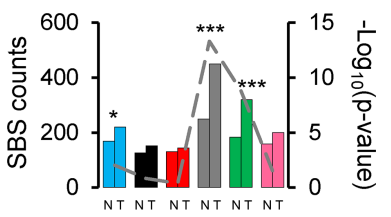

घ:G>A:T $\|\mathrm{C}: \mathrm{G}>\mathrm{T}: \mathrm{A}\| \mathrm{T}: \mathrm{A}>\mathrm{A}: \mathrm{T}$

- $C: G>C: G \square T: A>C: G \square T: A>G: C$

E
B
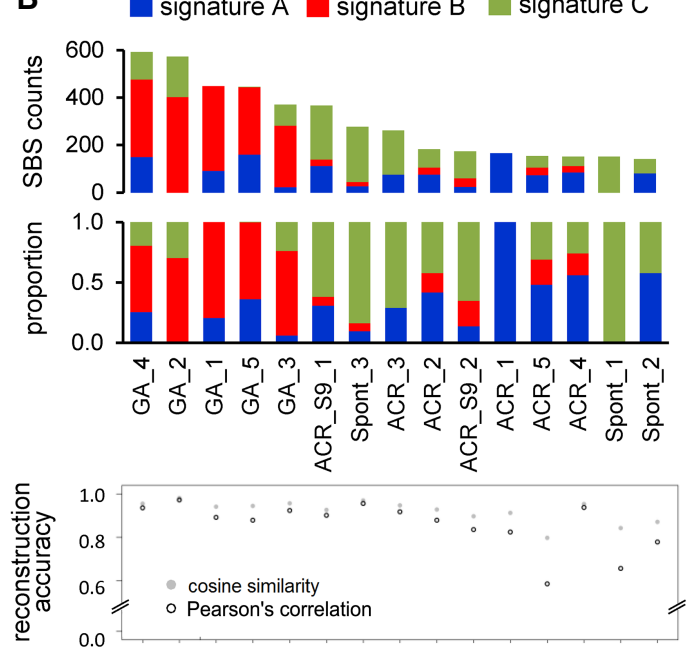

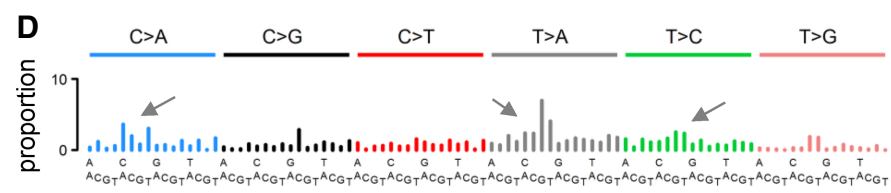<smiles>NC(=O)C(O)Cn1cnc2nc(N)[nH]c(=O)c21</smiles><smiles>CC(C)(C)ONc1ncn(CC(O)C(N)=O)c2ncnc1-2</smiles><smiles>C1CCC(C2CC3CC32)C1</smiles><smiles>CC(C)CCO</smiles>

$\sum_{\frac{\pi}{0}}^{0}$ , IS

IS

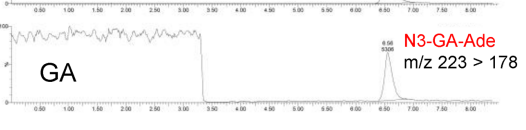

retention time $(\mathrm{min})$
F

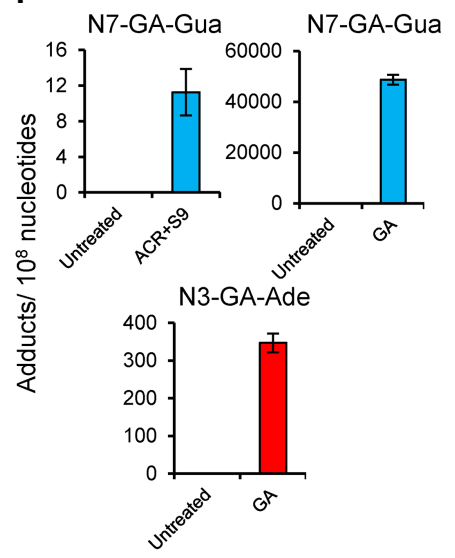

Figure 1. Analysis of the mutation patterns derived from experimental exome sequencing data. (A) Principle component analysis (PCA) of WES data. PCA was computed using as input the mutation count matrix of the clones that immortalized spontaneously (Spont) or were derived from exposure to acrylamide (ACR) or glycidamide (GA). Each sample is plotted considering the value of the first and second principal components (Dim1 and Dim2). The percentage of variance explained by each component is indicated within brackets on each axis. Spont and ACR- and GA-exposed samples are represented by differently colored symbols. (B) Mutational signatures ( $\operatorname{sig} A$, sig B, and sig C), identified by NMF, and their contribution to each sample ( $x$-axis), assigned either by absolute SBS counts or by proportion (bar graphs). The reconstruction accuracy of the identified mutational signatures in individual samples is shown in the bottom dot plot ( $y$-axis value of $1=100 \%$ accuracy). (C) Transcription strand bias analysis for the six mutation types in GA-exposed clones. For each mutation type, the number of mutations occurring on the transcribed $(\mathrm{T})$ and nontranscribed $(\mathrm{N})$ strand is shown on the $y$-axis. $\left.{ }^{* * *}\right) P<10^{-8}$, $\left(^{*}\right) P<10^{-2}$. (D) Extraction of GA signature, with arrows pointing at the enriched SBS classes. The contribution of signature 17 (T:A $>$ G:C in 5'-NTT-3' context), present in all clones, was decreased by performing NMF on human-TP53 knock-in (Hupki) MEF samples pooled with primary tumor samples with high levels of signature 17 (see Methods and Supplemental Methods). (E) DNA adducts analysis as determined by LC-MS/MS. (F) Levels of N7-GAGua adduct in ACR + S9- and GA-treated cells and N3-GA-Ade DNA adduct level in GA-treated cells compared with untreated cells yielding no adducts. The data are presented as the number of adducts in $10^{8}$ nucleotides in replicated experiments $(n \geq 2)$.

\section{Comparison of the GA signature with PCAWG mutational signatures}

We next performed cosine similarity comparison of the putative GA signature with the recently updated PCAWG SBS mutational signatures (Alexandrov et al. 2018) and with known T:A>A:Trich experimental signatures (Fig. 2A; Supplemental Figs. S7, S9). The highest cosine similarity value $(84 \%)$ corresponded to PCAWG SBS25 (Fig. 2A). However, unlike the GA signature, neither SBS25 nor any other signatures show TSB for the three

\section{Genome Research}

www.genome.org 


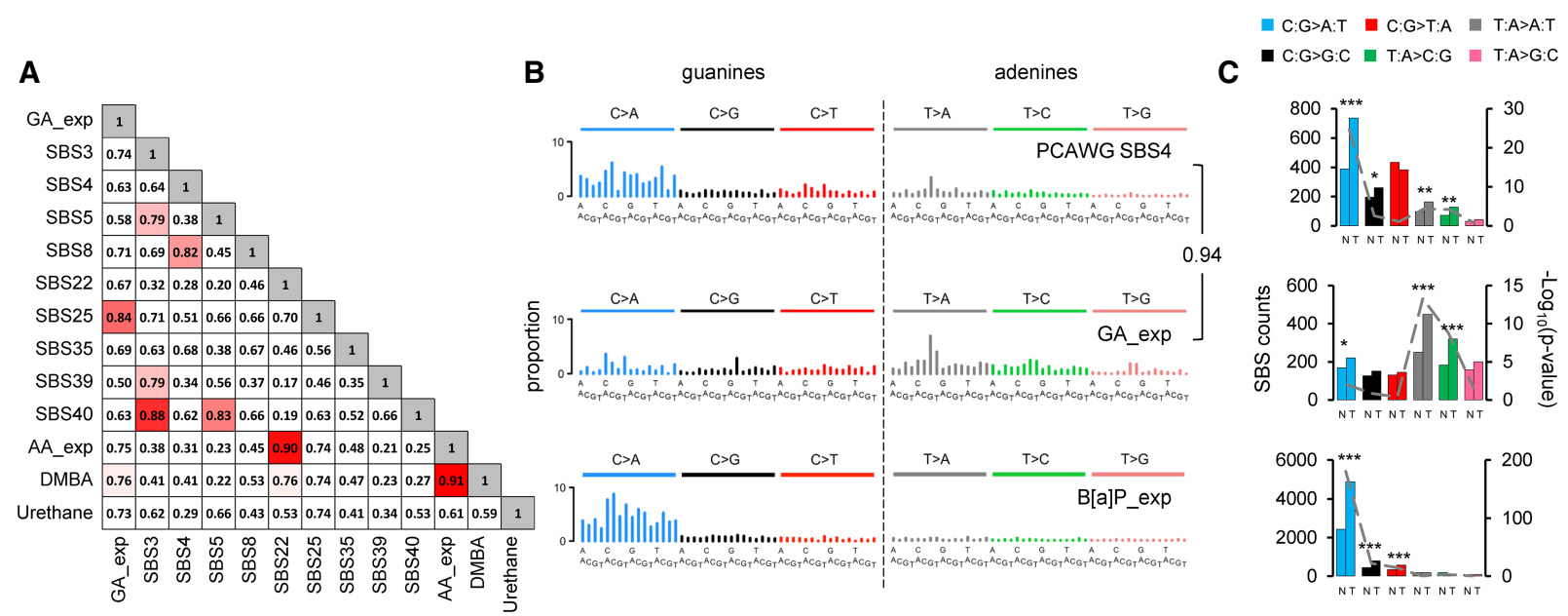

Figure 2. Comparison of GA signature to known signatures. (A) Cosine similarity matrix comparing GA mutational signature with the human PCAWG data (SBS3, $-4,-5,-8,-22,-25,-35,-39$, and -40 ) and other A $>$ T-rich mutational signatures from experimental exposure assays using specific carcinogens (7,12-dimethylbenz $[a]$ anthracene [DMBA], urethane, and aristolochic acid [AA]). (B) Comparison of PCAWG SBS4 with two experimentally derived signatures: $\mathrm{B}[\mathrm{a}] \mathrm{P} \_$exp = benzo[a]pyrene mutational signature extracted from HMECs; GA_exp=GA mutational signature extracted from MEF cells. Cosine similarity between the $\mathrm{T}>\mathrm{N}$ (adenine) components of SBS4 and GA signature is shown on the right. (C) Transcription strand bias analysis for the six mutation types underlying the signatures in panel $B$. For each mutation type (using the pyrimidine convention), the number of mutations occurring on the transcribed $(T)$ and nontranscribed $(N)$ strand is shown on the left $y$-axis. The significance is expressed as $-\log _{10}(P$-value $)$ indicated on the right $y$-axis. $\left({ }^{* *}\right) P<10^{-8},(* *) P<10^{-4},(*) P<10^{-2}$.

mutation classes (C:G>A:T, T:A>A:T, and T:A>C:G). Thus, the mutation patterns with a three-class strand bias generated by the GA treatment render the resulting mutational signature unique and novel.

\section{GA signature in the human pan-cancer genomes}

The initial visual comparison with PCAWG signatures indicated similarity between the GA signature and signature SBS4 of tobacco smoking (Supplemental Fig. S8; Alexandrov et al. 2018), in keeping with the established presence of ACR in tobacco smoke. This was further corroborated by the cosine similarity of $94 \%$ between the adenine $(\mathrm{T}>\mathrm{N})$ components of SBS4 and the GA signature (Fig. 2B). We thus hypothesized that SBS4 reflects the coexposure to benzo[a]pyrene (B[a]P; generating the predominant, strandbiased $\mathrm{C}>\mathrm{N} /$ guanine mutations) and to $\mathrm{GA}$ (generating strandbiased $\mathrm{T}>\mathrm{N} /$ adenine mutations) (Fig. 2B,C; Supplemental Fig. S8). To obtain experimental evidence, we modeled a B[a]P mutational signature by whole-genome sequencing (WGS) of cell clones derived from $\mathrm{B}[\mathrm{a}] \mathrm{P}$-exposed normal human mammary epithelial cells (HMECs) (Stampfer and Bartley 1985, 1988). This yielded a robust pattern characterized by predominant strand-biased guanine (mainly C:G>A:T) mutation levels and negligibly mutated adenines (T > N) (Fig. 2B; Supplemental Figs. S8, S10; Supplemental Table S4). Next, we interrogated the PCAWG data for the presence of the experimentally defined, 192-class (strand-biased) GA and $\mathrm{B}$ [a]P signatures in 1584 tumors of 19 cancer types from 14 organ sites (Fig. 3; Supplemental Table S5). The stringency of the process was controlled by determining the $P$-value and the false-discovery rate (FDR) for the signature presence test and the reconstruction accuracy (Supplemental Table S6) and by modeling false-positive rates (FPRs) and FDRs of the experimental signature detection using 2000 synthetic tumors as described in the Methods and in Supplemental Tables S7 through S10. In the subset of PCAWG-7 cancers known to carry SBS4 signature (adenocarcinomas and squamous cell carcinomas of the lung, hepatocellular carcinomas of the liver and head, and neck squamous cell carcinomas), we compared the GA and B[a]P signatures to estimated levels of SBS4 and found that in the lung and head and neck cancers, a combination of the GA and B[a]P signatures accounted for very similar numbers of mutations as SBS4, suggesting that SBS4 represents combined and highly correlated exposure to GA and $\mathrm{B}[\mathrm{a}] \mathrm{P}$ (Fig. 3A). In contrast, we found more variability in the assignment of mutation numbers to GA and B[a]P versus SBS4 in liver cancers (Fig. 3), which may reflect a weaker relationship between GA and B[a]P exposure because of generally more complex exposure history in the liver. Successful reconstruction of SBS4 by the experimental 192-class (strand-biased) GA and B[a]P signatures in the lung and liver human tumors enabled correct assignment of the GA signature in a subset of 24 lung adenocarcinomas, 42 lung SCCs, and 239 liver tumors with a subset of 184 GA-positive HCCs lacking the B[a]P signature mutations (Fig. 3B; Supplemental Table S11). Moreover, we identified the GA signature in additional 15 cancer types without SBS4, including clear cell renal cell carcinoma (78 GA-positive of 111 analyzed tumors), papillary renal cell carcinoma (26 GA-positive out of 32), biliary adenocarcinoma (20 GA-positive out of 35), colorectal adenocarcinomas (24 GA-positive out of 60), stomach adenocarcinoma (17 GA-positive out of 75), bladder transitional cell carcinoma (six GA-positive out of 23), and uterine adenocarcinoma (10 GA-positive out of 51) (Fig. 3B,C). The signature assignments results for the 537 individual GA-positive PCAWG tumors are summarized by cancer type in the Supplemental Table S11.

\section{Discussion}

ACR and GA exposures induce an almost identical set of tumors in both mice and rats, providing a substantial argument for a GA-mediated tumorigenic effect of ACR (Beland et al. 2015). This is supported by further mechanistic studies showing that lung tissue from mice exposed to ACR and GA displays comparable DNA adduct patterns, as well as similar mutation frequencies in the $c I I$ transgene (Manjanatha et al. 2015). Similar observations were 
A
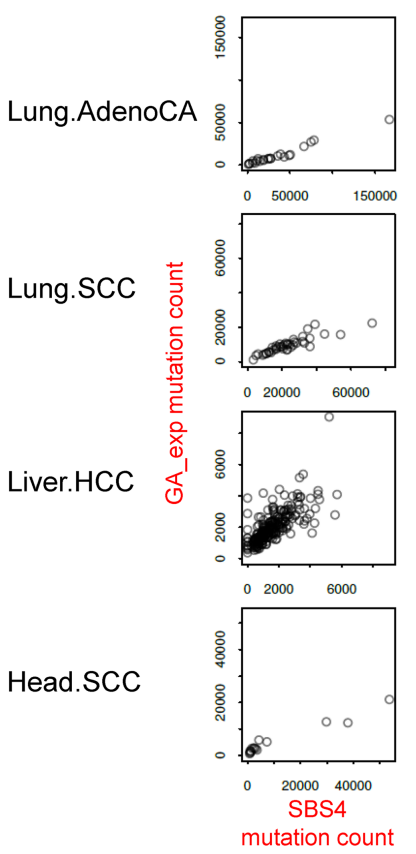

B

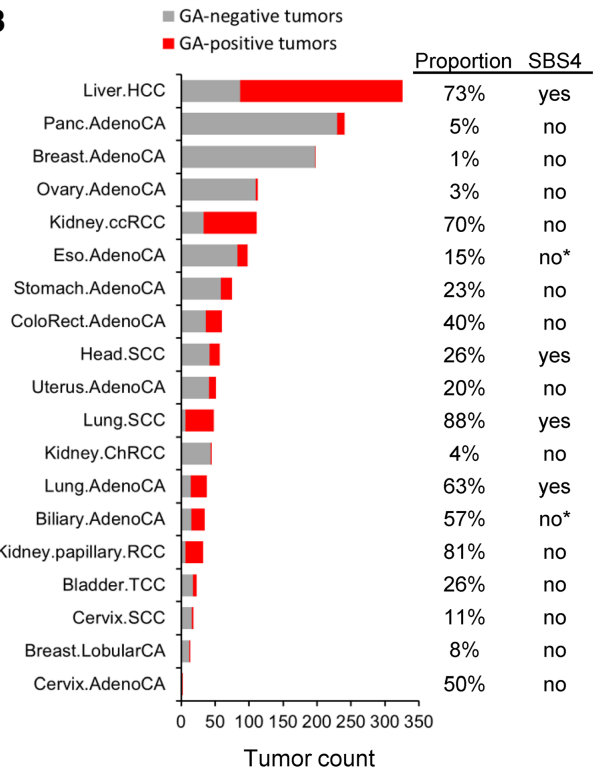

Liver.HCC

Head.SCC

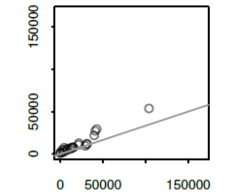

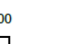
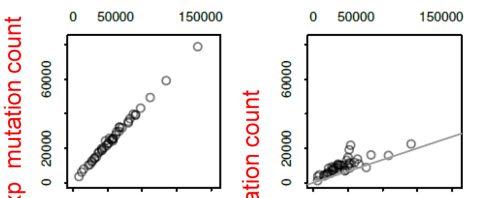

ब
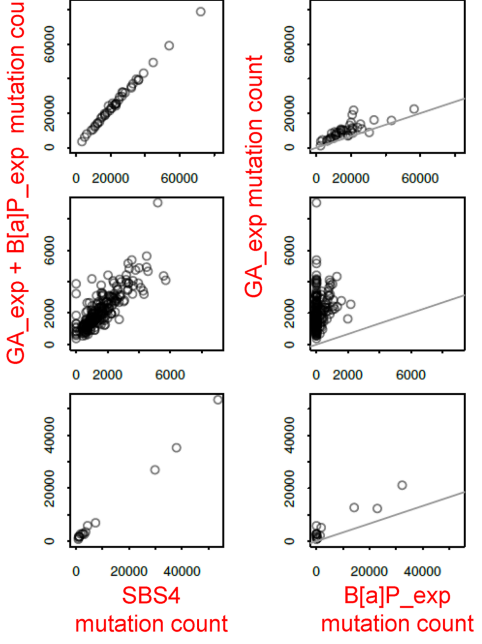

C

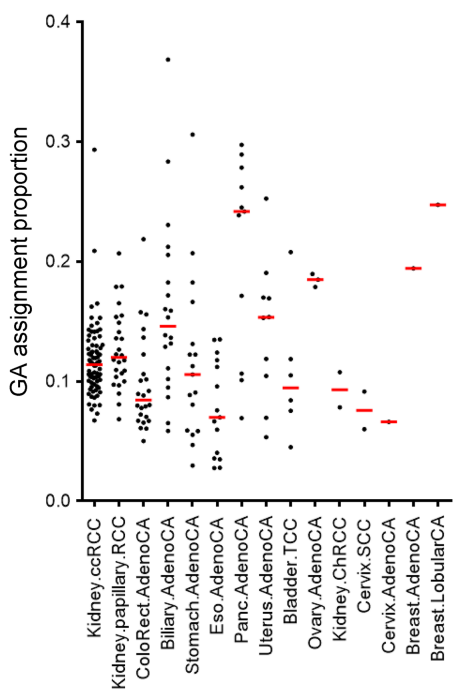

Figure 3. Identification of experimental GA signature in the human cancer PCAWG data sets. $(A)$ Scatter plots of the experimental GA_exp and B[a]P_exp mutational signature assignments by mSigAct show reconstruction of tobacco-smoking signature SBS4 assignments in cancer types with SBS4 present. (Lung.AdenoCA) Lung adenocarcinoma, (Lung.SCC) lung squamous cell carcinoma, (Liver.HCC) liver hepatocellular carcinoma (Head.SCC) head squamous cell carcinoma. The combination of GA_exp and B[a]P_exp mutation counts reconstructed SBS4 mutation counts in Lung.AdenoCA and Lung.SCC and, to an extent, in Head.SCC. In liver HCCs, GA counts alone partially reconstructed SBS4 mutation counts and indicate GA_exp-positive and B[a]P_exp-negative tumors (third row, right scatter plot). The lines in GA versus B[a]P scatter plots have a slope of 0.3 , reflecting the $3: 1$ ratio of $B[a] P: G A$ mutation counts that reconstruct SBS4. (B) Summary of GA mutation assignment analysis of 1584 individual tumors of 19 cancer types from the PCAWG data sets. Assignments were performed using mSigAct (positivity was determined by the signature.presence.test tool at FDR $<0.05$ ) with the PCAWG annotations of signature present in each subtype, in addition to the GA and $\mathrm{B}[\mathrm{a}] \mathrm{P}$ signatures. The tumor types manifesting or lacking SBS4 signature of tobacco smoking are labeled accordingly in the column SBS4. Asterisk denotes borderline SBS4 presence in PCAWG Billiary.AdenoCA (two of 173, 1.16\%) and Eso.AdenoCA (two of 347, 0.06\%). Proportion indicates percentage of GA-positive tumors within each listed cancer type. (C) The dot plot shows the proportion of mutations assigned to GA signature among other identified signatures (see Supplemental Material) in individual tumors of cancer types not showing the direct effects of tobacco smoking (i.e., lacking signature SBS4). Red horizontal lines denote median values ( $y$-axis, $1=100 \%)$.

made in the context of in vitro mutagenicity of ACR in human and mouse cells, suggesting the key role for the epoxide metabolite GA to form premutagenic DNA adducts (Besaratinia and Pfeifer 2004). Thus, in keeping with the established ACR/GA carcinogenicity in rodents (IARC 1994; Olstørn et al. 2007; Von Tungeln et al. 2012; Beland et al. 2015), our findings provide new information on the characteristic mutagenic effects of GA and their contribution to tumor development.

The observation that ACR itself is not efficiently metabolized by MEFs is consistent with similar differences reported by previous animal carcinogenicity studies. In neonatal B6C3F1 mice, GA, but not ACR, induces hepatocellular carcinomas, likely because of the inability of neonatal mice to efficiently metabolize ACR (Von Tungeln et al. 2012). Moreover, unlike ACR, GA induces tumors in the small intestine in a dosedependent manner upon perinatal exposure (Olstørn et al. 2007). Similar differences between GA and ACR mutagenicity, possibly because of limited metabolization of ACR, were observed in vitro (Besaratinia and Pfeifer 2004). We addressed the lack of ACR activation by the addition of human $S 9$ fraction, yet the assessment of DNA adducts suggested limited metabolic activation of ACR with adduct levels substantially lower compared with the direct GA exposure. This may explain the mutagenicity differences observed between GA and ACR. A consistent minor contribution of the GA mutational signature was detected in the majority of ACR clones, whereas it was mostly absent in the Spont clones, suggesting subtle metabolic activation of ACR in the MEFs resulting in low levels of GA. However, a robust mutational signature in the experimental setting was generated exclusively by exposing the cells directly to GA.

Single reporter gene studies had previously linked ACR and GA exposure to multiple different mutation types. Thanks to the larger number of mutations obtained by exome sequencing, we were able to attribute to the GA exposure a particular mutational signature characterized by three strand-biased mutation classes (C:G>A:T, T:A>A:T, and $\mathrm{T}: \mathrm{A}>\mathrm{C}: \mathrm{G})$. The identification of the N7GA-Gua and N3-GA-Ade DNA adducts originating from the metabolic conversion of ACR (Segerbäck et al. 1995; da Costa et al. 2003; Besaratinia and Pfeifer

\section{Genome Research}

www.genome.org 
2005), underlines the relationship between DNA adduct profiles and the mutational signature of GA. N3-GA-Ade and N7-GA-Gua are depurinating adducts resulting in apurinic/apyrimidinic sites. During replication, these lead to misincorporation of deoxyadenine, leading to the respective T:A $>A: T$ and $C: G>A: T$ transversions observed in the GA signature. The $T: A>C: G$ transitions enriched in the GA signature correspond to the miscoding N1-GA-Ade adduct, the most commonly identified adenine adduct in vitro (Randall et al. 1987; da Costa et al. 2003; Besaratinia and Pfeifer 2005; Ishii et al. 2015). The levels of the guanine adduct were especially high in the GA-exposed MEF cells, whereas the associated $\mathrm{C}: \mathrm{G}>\mathrm{A}: \mathrm{T}$ transversions in the resulting postsenescence clones were less represented. This could reflect differences in DNA repair efficiency concerning the individual guanine and adenine adduct species or the fact that the resulting clones are derived from single cells that selectively immortalized but do not accurately represent the bulk exposed primary cell population in which the GA-DNA adduct levels were measured after exposure. It is also plausible that the excessive and possibly highly cytotoxic N7GA-Gua adduct burden leads to negative selection of a large number of affected cells.

The established animal models (Beland et al. 2013, 2015) of ACR- and GA-mediated tumorigenesis provide a suitable starting point for a comparison of the mutational signatures obtained from the mouse and in vitro. Next, genome-scale sequencing of human tumors and adduct analysis of normal tissues collected in well-designed molecular epidemiological studies focusing on ACR intake are warranted to provide further evidence that the GA signature mutations identified in various cancer types indeed correlate with the exposure to ACR.

The GA signature has not been identified among the currently known computationally extracted PCAWG signatures (Fig. 2A; Alexandrov et al. 2018). Here we show that a new pattern can be identified in a large subset of pan-cancer tumors when experimentally modeled signatures are combined with sophisticated computational signature reconstruction methods while considering the extended features, such as TSB supported by premutagenic adduct analysis. Such integrated approaches can thus lead to future identification of yet unrecognized carcinogen signatures that may be eluding the solely computation-based analyses of the pan-cancer data.

The quest for understanding the contribution of ACR to cancer development is reflected by recent accumulation of mechanistic data on the compound's mutagenicity and carcinogenicity in experimental models. The possibly carcinogenic effects of ACR in humans were recommended for re-evaluation by the Advisory Group to the Monographs Program of the International Agency for Research on Cancer (Straif et al. 2014). Our findings related to the reconstruction of signature SBS4 by the experimental signatures of $\mathrm{GA}$ and $\mathrm{B}[\mathrm{a}] \mathrm{P}$, together with the detection of the GA signature in lung and liver cancer, are relevant given the established high content of ACR in tobacco smoke. Compared with the GA effects, experimental $\mathrm{B}[\mathrm{a}] \mathrm{P}$ exposure generates very few $\mathrm{T}>\mathrm{N}$ (adenine) mutations. However, we cannot exclude a possibility that in the human tissues directly exposed to tobacco smoke the adenine residues can be targeted by carcinogens such as $\mathrm{B}[\mathrm{a}] \mathrm{P}$ derivatives or nitrosamines.

A subset of 184 liver tumor samples identified in this study harbored the GA signature but no features of the $\mathrm{B}[\mathrm{a}] \mathrm{P}$ signature or SBS4 (Fig. 3B; Supplemental Material). Furthermore, we found 217 GA-positive, SBS4-negative tumors of additional 15 cancer types (Fig. 3B,C). The numerous GA-positive, SBS4-negative tu- mors are of particular interest as they likely reflect dietary and/or occupational exposures to ACR unrelated to tobacco smoking. Overall, our findings offer new insights into the thus-far tenuous association of ACR with human carcinogenesis.

\section{Methods}

\section{Source and authentication of primary cells}

Primary human-p53 knock-in (Hupki) MEFs were isolated from 13.5-d-old Trp53 $3^{\text {tm/Holl }}$ mouse embryos from the Central Animal Laboratory of the Deutsches Krebsforschungszentrum as described previously (Liu et al. 2004). The mice had been tested for specific pathogen-free (SPF) status. The derived primary cells were genotyped for the human TP53 codon 72 polymorphism (Table 1 ) to authenticate the embryo of origin. Cells from three different embryos (E210, E213, and E214) were used for the exposure experiments (Table 1). All subsequent cell cultures were routinely tested at all stages for the absence of mycoplasma.

\section{Cell culture, exposure, and immortalization}

The primary MEF cells were expanded in advanced DMEM supplemented with $15 \%$ fetal calf serum, $1 \%$ penicillin/streptomycin, $1 \%$ pyruvate, $1 \%$ glutamine, and $0.1 \% \beta$-mercaptoethanol. The cells were then seeded in six-well plates and, at passage 2 , were exposed for $24 \mathrm{~h}$ to $5 \mathrm{mM}$ ACR (A4058, Sigma-Aldrich) in the presence of $2 \%$ human $S 9$ fraction (Life Technologies) complemented with NADPH (Sigma-Aldrich) or the absence of S9 to $10 \mathrm{mM}$ ACR or 3 mM GA (04704, Sigma-Aldrich), or to vehicle (PBS). Exposed and untreated control primary cells were cultured until they bypassed senescence and immortalized clonal cell populations could be isolated (Todaro and Green 1963). The HMEC cultures used in this study for WGS were generated from primary HMECs (passage 4) exposed to $\mathrm{B}[\mathrm{a}] \mathrm{P}$ and propagated in M87A medium to passage 13, as described previously (Stampfer and Bartley 1985, 1988; Garbe et al. 2009; Severson et al. 2014).

\section{MTT assay for cell metabolic activity and viability}

Cells were seeded in 96-well plates and treated as indicated. Cell viability was measured $48 \mathrm{~h}$ after treatment cessation using the CellTiter $96 \mathrm{AQ}_{\text {ueous }}$ One Solution Cell Proliferation Assay (Promega). Plates were incubated for $4 \mathrm{~h}$ at $37^{\circ} \mathrm{C}$, and absorbance was measured at $492 \mathrm{~nm}$ using the Apollo $11 \mathrm{LB} 913$ plate reader. The MTT assay was performed in triplicate for each experimental condition.

\section{Phospho-H2AFX immunofluorescence}

Immunofluorescence staining of phosphorylated histone H2AFX $(\gamma \mathrm{H} 2 \mathrm{AFX})$ was performed using phospho-histone H2A.X (Ser139) (20E3) Rabbit monoclonal antibody (9718, Cell Signaling Technology). Briefly, primary MEFs were seeded on coverslips in 12-well plates and, the following day, treated as indicated in duplicate for $24 \mathrm{~h}$. Four hours after treatment cessation, the cells were fixed with $4 \%$ formaldehyde for $15 \mathrm{~min}$ at room temperature. Following blocking in 5\% normal goat serum (31872, Life Technologies) for $60 \mathrm{~min}$, they were incubated with the $\gamma \mathrm{H} 2 \mathrm{AFX}$-antibody (1:500 in $1 \% \mathrm{BSA}$ ) overnight at $4^{\circ} \mathrm{C}$. Subsequent incubation with a fluorochrome-conjugated secondary antibody (4412, Cell Signaling Technology) was performed for $60 \mathrm{~min}$ at room temperature. Coverslips were mounted in Vectashield mounting medium with DAPI (Eurobio). Immunofluorescence images were captured using a Nikon Eclipse Ti. 


\section{DNA adduct analysis}

GA-DNA adducts (N7-GA-Gua and N3-GA-Ade) were quantified by LC-MS/MS with stable isotope dilution as previously described (da Costa et al. 2003). The DNA was isolated from the cells using standard digestion with Proteinase $\mathrm{K}$, followed by phenol-chloroform extraction and ethanol precipitation. The DNA was subsequently treated with RNase A and T1, extracted with phenol-chloroform, and reprecipitated with ethanol. N7-GA-Gua and N3-GA-Ade were released by neutral thermal hydrolysis for $15 \mathrm{~min}$, using Eppendorf Thermomixer R (Eppendorf North America) set to $99^{\circ} \mathrm{C}$. The samples were filtered through Amicon $3 \mathrm{~K}$ molecularweight cutoff filters (Merck Millipore) to separate the adducts from the intact DNA. The LC-MS/MS used for quantification consisted of an Acquity UPLC system (Waters) and a Xevo TQ-S triple quadrupole mass spectrometer (Waters). The following MRM transitions were monitored with a cone voltage of $50 \mathrm{~V}$ and a collision energy of $20 \mathrm{eV}$ : N3-GA-Ade, m/z 223 $\rightarrow 178$; [15N5]N3-GA-Ade (internal standard), m/z 228 $\rightarrow 183$; N7-GA-Gua, m/z 239 $\rightarrow 152$; and [15N5]N7-GA-Gua (internal standard), m/z 244 $\rightarrow 157$ (da Costa et al. 2003).

\section{TP53 genotyping}

Exons 4 to 8 of the knocked-in human TP53 gene (NC_000017.11) were sequenced using standard protocols. Sanger sequencing of PCR products was performed at BIOfidal, using the Applied Biosystems 3730xl genetic analyzer. The amplicon and sequencing primers are listed in the Supplemental Methods. Sequences were analyzed using the CodonCode Aligner version 7.1 software.

\section{Library preparation and WES}

Refer to the online Supplemental Methods for details on the standard procedures for library preparation and WES, sequencing data preprocessing, read alignment, and the calling of the SBS and indel variants in the MEF and HMEC cell lines.

\section{Bioinformatics and extraction of experimental mutational signatures}

Refer to the Supplemental Methods for detailed information on PCA, assessment of sequencing-related artifacts and damage, and computation of the TSB and its significance. The TSB was considered statistically significant at $P$-value $\leq 0.05$. To analyze the mutation spectra and treatment-specific mutational signatures, filtered mutations were classified into 96 types corresponding to the six possible base substitutions (C:G>A:T, C:G>G:C, C:G>T:A, T:A> A:T, T:A >C:G, T:A>G:C) and the 16 combinations of flanking nucleotides immediately $5^{\prime}$ and $3^{\prime}$ of the mutated base. Mutation patterns were then deconvolved into mutational signatures using NMF (Brunet et al. 2004; Alexandrov et al. 2013b) embedded in the MutSpec suite (Ardin et al. 2016). For details on estimates of the optimal number of signatures to extract, see the Supplemental Methods. The reconstruction error calculation evaluated the accuracy with which the deciphered mutational signatures describe the original mutation spectra of each sample by applying Pearson's correlation and cosine similarity.

The GA mutational signature was further polished by using an extended input including samples from ICGC (ESAD-UK study) with high level of signature 17 ( $>65 \%$ contribution as determined by independent NMF analysis), and with samples from the TCGA esophageal adenocarcinoma (ESCA) and gastric cancer (STAD) collection (exon data, to address comparable coverage of the genome). The samples used for this procedure are listed in the
Supplemental Methods, and the results are summarized in Supplemental Figures S6 and S7.

Cosine similarity analysis was used to evaluate the concordance between the identified T:A > A:T-rich mutational signature of GA with the newly characterized SBS mutational signatures from the PCAWG (pan-cancer whole genome) data (Alexandrov et al. 2018). Cosine similarity values of more than 0.5 were found for PCAWG SBS3, SBS4, SBS5, SBS8, SBS22, SBS25, SBS35, SBS39, and SBS40 and the experimentally derived mutational signature of AA (Olivier et al. 2014; Ardin et al. 2016), 7,12-dimethylbenz [a]anthracene (DMBA) (McCreery et al. 2015; Nassar et al. 2015), and urethane (Westcott et al. 2014).

The experimental B[a]P signature was generated by WGS (using Illumina HiSeq X Ten by GENEWIZ) of finite lifespan poststasis clones derived from primary HMECs treated with $\mathrm{B}[\mathrm{a}] \mathrm{P}$, as previously described (Stampfer and Bartley 1985, 1988; Severson et al. 2014). Following read alignment to NCBI GRCh38 genome build, mutations were called in the two poststasis samples with MuTect2 or Strelka2.8 using a primary HMEC sample as a comparison. Only mutations called by both algorithms were retained, and additional criteria were applied to filter out mutations with a match in public SNP databases (dbSNP150, and/or AF >0.001 in either 1000 Genomes, gnomAD or NHLBI-ESP), with an allele frequency above zero in the primary sample, with coverage lower than 10 reads, or mutations overlapping tandem repeats. Finally, a cut-off was applied on VAF, and only mutations with a VAF equal or higher than $20 \%$ were retained, being 54,587 unique mutations. The NMF procedure to extract the experimental $\mathrm{B}[\mathrm{a}] \mathrm{P}$ signature used input extended with SBS data from the TCGA lung cancer collection (15 Lung.AdenoCA positive [ $>50 \%$ ] for tobacco-smoking SBS4, 15 Lung.AdenoCA negative for SBS4, 15 Lung.SCC positive [ $>50 \%]$ for SBS4 and 15 Lung.SCC negative for SBS4). See the Supplemental Methods for sample details. The recovered signatures showed the strongest enrichment of the C>A-based signature B (Supplemental Fig. S10) in the B[a]P-treated HMEC clones. We next calculated the reconstruction error to evaluate the accuracy with which the extracted $\mathrm{B}[\mathrm{a}] \mathrm{P} \_$exp signature describes the original mutation spectra of each sample by applying Pearson's correlation and cosine similarity (Supplemental Fig. S10).

\section{Identification of the experimental signatures in PCAWG data}

We used the mutational signature activity (mSigAct v0.10.R) software (Ng et al. 2017) to test for the presence of the experimental mutational signatures of GA and $\mathrm{B}[\mathrm{a}] \mathrm{P}$ in the human primary tumor data from PCAWG study. mSigAct conducts a statistical test for optimal reconstructions of the observed human tumor mutation spectrum with and without the GA mutational signature, in addition to a set of other mutational signatures from the PCAWG study. The 192-class strand-biased versions of the GA and $\mathrm{B}[\mathrm{a}] \mathrm{P}$ mutational signatures (Supplemental Fig. S8; Supplemental Table S4) were used to detect tumors with the experimentally defined signatures present, at high stringency achieved also by incorporating the same TSB information in the 192-class reconstructions of each tumor. To generate a 192-class reconstructed spectrum, the assignment of mutation counts for each 192-class signature is determined by mSigAct and multiplied with the 192-class versions of the PCAWG, GA, and B[a]P mutational signatures. The 192-class versions of each signature and spectrum is equivalent to the 96-class versions when the mutation counts on each strand are summed and then represented in the pyrimidine mutations (C > A, C > G, C > T, T > A, T > C, T > G). Specifically, B[a]P was added to cancer types with tobacco-smoking SBS4 signature previously found in the PCAWG signature set, and a combination of $\mathrm{B}[\mathrm{a}] \mathrm{P}$ and GA signatures was used in these cancers to reconstruct SBS4.

\section{Genome Research}

www.genome.org 
For other signatures and cancers without evidence of SBS4 present, only GA was used to reconstruct the tumor spectra. This was followed by computing the likelihood ratio test between the original spectrum and the reconstructed tumor. A total of 1673 tumor samples from the PCAWG repository from 20 cancer subtypes were interrogated. We excluded hypermutated and recently identified AA signature-containing tumors ( $\mathrm{Ng}$ et al. 2017) as the presence of strong $\mathrm{T}>\mathrm{A}$ signature adversely affected the reconstruction process. A set of active mutational signatures were obtained from the PCAWG annotations of each cancer subtype, with flat signatures (SBS3, SBS8) removed to improve the sparsity of the mutation assignments. Final assignments of mutations to each mutational signature were performed by using the 96-class mutational signatures. Further fine-tuning was conducted using parameters for a negative binomial model, and the FDR was adjusted for mutational signature presence (FDR $<0.05$ ).

The proportion matrices of the strand-biased and NMF versions of the experimental GA signature, the GA signature normalized to the human genome trinucleotide frequency to allow for human PCAWG data screening, and the strand-biased and NMF versions of the whole-genome $\mathrm{B}[\mathrm{a}] \mathrm{P}$ signature are available in Supplemental Table S4. The statistics underlying the assignment of GA_exp to PCAWG cancer data sets ( $P$-values for "signature.presence.test" and cosine similarity between the reconstruction and spectra) are summarized in Supplemental Table S5.

\section{FPR and FDR estimation for GA signature detection in synthetic tumors}

To determine how often false positives arise when detecting the GA signature with mSigAct and to accurately estimate the FDR of the detection of GA signature, we performed a deeper validation analysis. We generated 2000 synthetic tumors with signatures from the PCAWG-7 data set and assignments sampled from the assignments to each signature in the PCAWG-7 data set, which represented the tumor types in which we found GA signature present, with similar signatures and mutation burdens associated with each signature. The synthetic tumors had the same frequency of observing a particular signature for a cancer type, similar to the PCAWG-7 tumors. One hundred tumors per 20 tumor types (included in the main analysis and listed in Supplemental Table S9) have been generated, with 1015 of the tumors harboring GA signature and 985 with GA signature absent. By using the synthetic tumor set and mSigAct to assign GA signature, we established the true-positive rates (TPRs), FPRs and FDRs (calculated by using the raw synthetic tumor counts and the formula $\mathrm{FP} /(\mathrm{TP}+\mathrm{FP}))$. The results are shown as a short summary (Supplemental Table S7), raw tumor counts (Supplemental Table S8), per cancer type distribution (Supplemental Table S9) and a full listing of TPRs, FPRs, and FDRs (Supplemental Table S10).

\section{Data access}

Aligned WES reads from the primary MEF cells and clones arising from ACR- and GA-treated cultures and immortalized spontaneously, as well as Sanger sequencing files, have been submitted to the NCBI BioProject database (BioProject; https://www.ncbi.nlm .nih.gov/bioproject) under accession number PRJNA238303 (for the individual BioSample accession numbers, refer to Supplemental Tables S12, S13). The WES data reported here are a new extension of the BioProject PRJNA238303 dedicated to systematic identification of mutational signatures of carcinogenic agents (Olivier et al. 2014).

\section{Acknowledgments}

We thank the New York University Genome Technology Center, funded in part by the NIH/NCI Cancer Center support grant P30CA016087, and GENEWIZ, for expert assistance with Illumina sequencing. The study was supported by funding obtained from INCa-INSERM (Plan Cancer 2015 grant to J.Z.), NIH/NIEHS (1R03ES025023-01A1 grant to M.O.), and the Singapore National Medical Research Council (NMRC/CIRG/1422/2015 grant to S.G.R.) and the Singapore Ministry of Health via the Duke-NUS Signature Research Programmes to S.G.R. M.R.S. was supported by the U.S. Department of Energy under contract no. DE-AC0205CH11231. M.P. was supported by the European Commission FP7 Marie Curie Actions-People-COFUND Fellowship. The views expressed in this article do not necessarily represent those of the U.S. Food and Drug Administration.

Author contributions: M.Z., M.K., and J.Z. drafted the manuscript and prepared figures. M.I.C. and F.A.B. performed DNA adduct analyses. M.Z., M.P., S.V., and M.K. performed laboratory studies under the supervision of M.R.S., J.M., A.H., M.H., and J.Z.; M.Z., A.W.T.N, M.A., C.R., V.C., A.R., L.B., M.O., and S.R.G. performed computational analyses and prepared relevant display items. M.Z., A.W.T.N., A.H., K.G., J.M., M.O., F.A.B., M.K., and J.Z. edited the manuscript, and M.Z., K.G., M.H., M.K., and J.Z. designed the study.

\section{References}

Alexandrov LB, Nik-Zainal S, Wedge DC, Aparicio SAJR, Behjati S, Biankin AV, Bignell GR, Bolli N, Borg A, Børresen-Dale A-L, et al. 2013a. Signatures of mutational processes in human cancer. Nature 500: 415421. doi:10.1038/nature 12477

Alexandrov LB, Nik-Zainal S, Wedge DC, Campbell PJ, Stratton MR. 2013b. Deciphering signatures of mutational processes operative in human cancer. Cell Rep 3: 246-259. doi:10.1016/j.celrep.2012.12.008

Alexandrov LB, Kim J, Haradhvala NJ, Huang MN, Ng AWT, Boot A, Covington KR, Gordenin DA, Bergstrom E, Lopez-Bigas N, et al. 2018. The repertoire of mutational signatures in human cancer. bioRxiv doi: $10.1101 / 322859$

Ardin M, Cahais V, Castells X, Bouaoun L, Byrnes G, Herceg Z, Zavadil J, Olivier M. 2016. MutSpec: a Galaxy toolbox for streamlined analyses of somatic mutation spectra in human and mouse cancer genomes. BMC Bioinformatics 17: 170. doi:10.1186/s12859-016-1011-z

Behjati S, Huch M, van Boxtel R, Karthaus W, Wedge DC, Tamuri AU, Martincorena I, Petljak M, Alexandrov LB, Gundem G, et al. 2014. Genome sequencing of normal cells reveals developmental lineages and mutational processes. Nature 513: 422-425. doi:10.1038/ nature 13448

Beland FA, Mellick PW, Olson GR, Mendoza MCB, Marques MM, Doerge DR. 2013. Carcinogenicity of acrylamide in $\mathrm{B} 6 \mathrm{C}_{3} \mathrm{~F}_{1}$ mice and $\mathrm{F} 344 / \mathrm{N}$ rats from a 2-year drinking water exposure. Food Chem Toxicol 51: 149-159. doi:10.1016/j.fct.2012.09.017

Beland FA, Olson GR, Mendoza MCB, Marques MM, Doerge DR. 2015. Carcinogenicity of glycidamide in $\mathrm{B}_{6} \mathrm{C}_{3} \mathrm{~F}_{1}$ mice and $\mathrm{F} 344 / \mathrm{N}$ rats from a two-year drinking water exposure. Food Chem Toxicol 86: 104-115. doi:10.1016/j.fct.2015.09.017

Besaratinia A, Pfeifer GP. 2003. Weak yet distinct mutagenicity of acrylamide in mammalian cells. J Natl Cancer Inst 95: 889-896. doi:10.1093/ jnci/95.12.889

Besaratinia A, Pfeifer GP. 2004. Genotoxicity of acrylamide and glycidamide. J Natl Cancer Inst 96: 1023-1029. doi:10.1093/jnci/djh186

Besaratinia A, Pfeifer GP. 2005. DNA adduction and mutagenic properties of acrylamide. Mutat Res 580: 31-40. doi:10.1016/j.mrgentox.2004.10 .011

Brunet JP, Tamayo P, Golub TR, Mesirov JP. 2004. Metagenes and molecular pattern discovery using matrix factorization. Proc Natl Acad Sci 101: 4164-4169. doi:10.1073/pnas.0308531101

Chawanthayatham S, Valentine CC 3rd, Fedeles BI, Fox EJ, Loeb LA, Levine SS, Slocum SL, Wogan GN, Croy RG, Essigmann JM. 2017. Mutational spectra of aflatoxin $B_{1}$ in vivo establish biomarkers of exposure for human hepatocellular carcinoma. Proc Natl Acad Sci 114: E3101-E3109. doi:10.1073/pnas.1700759114

da Costa GG, Churchwell MI, Hamilton LP, Von Tungeln LS, Beland FA, Marques MM, Doerge DR. 2003. DNA adduct formation from 
acrylamide via conversion to glycidamide in adult and neonatal mice. Chem Res Toxicol 16: 1328-1337. doi:10.1021/tx034108e

Garbe JC, Bhattacharya S, Merchant B, Bassett E, Swisshelm K, Feiler HS Wyrobek AJ, Stampfer MR. 2009. Molecular distinctions between stasis and telomere attrition senescence barriers shown by long-term culture of normal human mammary epithelial cells. Cancer Res 69: 75577568. doi:10.1158/0008-5472.CAN-09-0270

Ghanayem BI, McDaniel LP, Churchwell MI, Twaddle NC, Snyder R, Fennell TR, Doerge DR. 2005. Role of CYP2E1 in the epoxidation of acrylamide to glycidamide and formation of DNA and hemoglobin adducts. Toxico Sci 88: 311-318. doi:10.1093/toxsci/kfi307

Hoang ML, Chen CH, Chen PC, Roberts NJ, Dickman KG, Yun BH, Turesky RJ, Pu YS, Vogelstein B, Papadopoulos N, et al. 2016. Aristolochic acid in the etiology of renal cell carcinoma. Cancer Epidemiol Biomarkers Prev 25 1600-1608. doi:10.1158/1055-9965.EPI-16-0219

Hogervorst JG, Schouten LJ, Konings EJ, Goldbohm RA, Brandt P. 2008. Dietary acrylamide intake and the risk of renal cell, bladder, and prostate cancer. Am J Clin Nutr 87: 1428-1438. doi:10.1093/ajcn/87.5.1428

Hollstein M, Alexandrov LB, Wild CP, Ardin M, Zavadil J. 2017. Base changes in tumour DNA have the power to reveal the causes and evolution of cancer. Oncogene 36: 158-167. doi:10.1038/onc.2016.192

Huang MN, Yu W, Teoh WW, Ardin M, Jusakul A, Ng AWT, Boot A, AbediArdekani B, Villar S, Myint SS, et al. 2017. Genome-scale mutational signatures of aflatoxin in cells, mice, and human tumors. Genome Res $\mathbf{2 7}$ 1475-1486. doi:10.1101/gr.220038.116

Huskova H, Ardin M, Weninger A, Vargova K, Barrin S, Villar S, Olivier M, Stopka T, Herceg Z, Hollstein M, et al. 2017. Modeling cancer driver events in vitro using barrier bypass-clonal expansion assays and massively parallel sequencing. Oncogene 36: 6041-6048. doi:10.1038/onc.2017 .215

International Agency for Research on Cancer (IARC). 1994. Some industrial chemicals: IARC monographs on the evaluation of carcinogenesis risks to humans, Vol. 60. World Health Organization Press, Geneva.

Ishii Y, Matsushita K, Kuroda K, Yokoo Y, Kijima A, Takasu S, Kodama Y, Nishikawa A, Umemura T. 2015. Acrylamide induces specific DNA adduct formation and gene mutations in a carcinogenic target site, the mouse lung. Mutagenesis 30: 227-235. doi:10.1093/mutage/geu062

Jelaković B, Castells X, Tomić K, Ardin M, Karanović S, Zavadil J. 2015. Renal cell carcinomas of chronic kidney disease patients harbor the mutational signature of carcinogenic aristolochic acid. Int J Cancer 136: 29672972. doi:10.1002/ijc.29338

Liu Z, Hergenhahn M, Schmeiser HH, Wogan GN, Hong A, Hollstein M. 2004. Human tumor p53 mutations are selected for in mouse embryonic fibroblasts harboring a humanized p53 gene. Proc Natl Acad Sci 101: 2963-2968. doi:10.1073/pnas.0308607101

Manjanatha MG, Guo LW, Shelton SD, Doerge DR. 2015. Acrylamide-induced carcinogenicity in mouse lung involves mutagenicity: cII gene mutations in the lung of big blue mice exposed to acrylamide and glycidamide for up to 4 weeks. Environ Mol Mutagen 56: 446-456. doi:10 $1002 / \mathrm{em} .21939$

McCreery MQ, Halliwill KD, Chin D, Delrosario R, Hirst G, Vuong P, Jen KY, Hewinson J, Adams DJ, Balmain A. 2015. Evolution of metastasis revealed by mutational landscapes of chemically induced skin cancers. Nat Med 21: 1514-1520, doi:10.1038/nm.3979

McFadden DG, Papagiannakopoulos T, Taylor-Weiner A, Stewart C, Carter SL, Cibulskis K, Bhutkar A, McKenna A, Dooley A, Vernon A, et al. 2014. Genetic and clonal dissection of murine small cell lung carcinoma progression by genome sequencing. Cell 156: 1298-1311. doi:10.1016/j .cell.2014.02.031

Meier B, Cooke SL, Weiss J, Bailly AP, Alexandrov LB, Marshall J, Raine K, Maddison M, Anderson E, Stratton MR, et al. 2014. C. elegans whole-genome sequencing reveals mutational signatures related to carcinogens and DNA repair deficiency. Genome Res 24: 1624-1636. doi:10.1101/ gr.175547.114

Milholland B, Dong X, Zhang L, Hao X, Suh Y, Vijg J. 2017. Differences between germline and somatic mutation rates in humans and mice. Nat Commun 8: 15183 . doi: $10.1038 /$ ncomms 15183

Mojska H, Gielecińska I, Cendrowski A. 2016. Acrylamide content in cigarette mainstream smoke and estimation of exposure to acrylamide from tobacco smoke in Poland. Ann Agric Environ Med 23: 456-461. doi:10.5604/12321966.1219187

Nassar D, Latil M, Boeckx B, Lambrechts D, Blanpain C. 2015. Genomic landscape of carcinogen-induced and genetically induced mouse skin squamous cell carcinoma. Nat Med 21: 946-954. doi:10.1038/nm.3878

Ng AWT, Poon SL, Huang MN, Lim JQ, Boot A, Yu W, Suzuki Y, Thangaraju S, Ng CCY, Tan P, et al. 2017. Aristolochic acids and their derivatives are widely implicated in liver cancers in Taiwan and throughout Asia. Sci Transl Med 9: eaan6446. doi:10.1126/scitranslmed.aan6446

Nik-Zainal S, Kucab JE, Morganella S, Glodzik D, Alexandrov LB, Arlt VM, Weninger A, Hollstein M, Stratton MR, Phillips DH. 2015. The genome as a record of environmental exposure. Mutagenesis 30: 763-770. doi:10 1093/mutage/gev073

Obón-Santacana M, Freisling H, Peeters PH, Lujan-Barroso L, Ferrari P, Boutron-Ruault MC, Mesrine S, Baglietto L, Turzanski-Fortner R, Katzke VA, et al. 2016a. Acrylamide and glycidamide hemoglobin adduct levels and endometrial cancer risk: a nested case-control study in nonsmoking postmenopausal women from the EPIC cohort. Int Cancer 138: 1129-1138. doi:10.1002/ijc.29853

Obón-Santacana M, Lujan-Barroso L, Freisling H, Cadeau C, Fagherazzi G, Boutron-Ruault M-C, Kaaks R, Fortner RT, Boeing H, Ramón Quirós J, et al. 2016b. Dietary and lifestyle determinants of acrylamide and glycidamide hemoglobin adducts in non-smoking postmenopausal women from the EPIC cohort. Eur J Nutr 56: 1157-1168. doi:10.1007/s00394016-1165-5

Obón-Santacana M, Lujan-Barroso L, Travis RC, Freisling H, Ferrari P, Severi G, Baglietto L, Boutron-Ruault MC, Fortner RT, Ose J, et al. 2016c. Acrylamide and glycidamide hemoglobin adducts and epithelial ovarian cancer: a nested case-control study in nonsmoking postmenopausal women from the EPIC cohort. Cancer Epidemiol Biomarkers Prev 25: 127 134. doi:10.1158/1055-9965.EPI-15-0822

Olesen PT, Olsen A, Frandsen H, Frederiksen K, Overvad K, Tjønneland A. 2008. Acrylamide exposure and incidence of breast cancer among postmenopausal women in the Danish Diet, Cancer and Health Study. Int J Cancer 122: 2094-2100. doi:10.1002/ijc.23359

Olivier M, Weninger A, Ardin M, Huskova H, Castells X, Vallée MP, McKay J, Nedelko T, Muehlbauer KR, Marusawa H, et al. 2014. Modelling mutational landscapes of human cancers in vitro. Sci Rep 4: 4482. doi:10 .1038/srep04482

Olstørn HBA, Paulsen JE, Alexander J. 2007. Effects of perinatal exposure to acrylamide and glycidamide on intestinal tumorigenesis in Min/+ mice and their wild-type litter mates. Anticancer Res 27: 3855-3864.

Pelucchi C, Bosetti C, Galeone C, La Vecchia C. 2015. Dietary acrylamide and cancer risk: an updated meta-analysis. Int J Cancer 136: 29122922. doi:10.1002/ijc.29339

Poon SL, Pang ST, McPherson JR, Yu W, Huang KK, Guan P, Weng WH, Siew EY, Liu Y, Heng HL, et al. 2013. Genome-wide mutational signatures of aristolochic acid and its application as a screening tool. Sci Transl Med 5: 197ra101-197ra101. doi:10.1126/scitranslmed.3006086

Randall SK, Eritja R, Kaplan BE, Petruska J, Goodman MF. 1987. Nucleotide insertion kinetics opposite abasic lesions in DNA. J Biol Chem 262: 6864-6870.

Scelo G, Riazalhosseini Y, Greger L, Letourneau L, Gonzàlez-Porta M, Wozniak MB, Bourgey M, Harnden P, Egevad L, Jackson SM, et al. 2014. Variation in genomic landscape of clear cell renal cell carcinoma across Europe. Nat Commun 5: 5135. doi:10.1038/ncomms6135

Secrier M, Li X, de Silva N, Eldridge MD, Contino G, Bornschein J, MacRae S, Grehan N, O'Donovan M, Miremadi A, et al. 2016. Mutational signatures in esophageal adenocarcinoma define etiologically distinct subgroups with therapeutic relevance. Nat Genet 48: 1131-1141. doi:10 1038/ng.3659

Segerbäck D, Calleman CJ, Schroeder JL, Costa LG, Faustman EM. 1995. Formation of N-7-(2-carbamoyl-2-hydroxyethyl) guanine in DNA of the mouse and the rat following intraperitoneal administration of $\left[{ }^{14} \mathrm{C}\right]$ acrylamide. Carcinogenesis 16: 1161-1165. doi:10.1093/carcin/16 .5 .1161

Severson PL, Vrba L, Stampfer MR, Futscher BW. 2014. Exome-wide mutation profile in benzo[a]pyrene-derived post-stasis and immortal human mammary epithelial cells. Mut Res Genet Toxicol Environ Mutagen 775776: 48-54. doi:10.1016/j.mrgentox.2014.10.011

Smith MT, Guyton KZ, Gibbons CF, Fritz JM, Portier CJ, Rusyn I, DeMarini DM, Caldwell JC, Kavlock RJ, Lambert PF, et al. 2016. Key characteristics of carcinogens as a basis for organizing data on mechanisms of carcinogenesis. Environ Health Perspect 124: 713-721. doi:10.1289/ehp .1509912

Stampfer MR, Bartley JC. 1985. Induction of transformation and continuous cell lines from normal human mammary epithelial cells after exposure to benzo[a]pyrene. Proc Natl Acad Sci 82: 2394-2398. doi:10.1073/ pnas.82.8.2394

Stampfer MR, Bartley JC. 1988. Human mammary epithelial cells in culture: differentiation and transformation. Cancer Treat Res 40: 1-24. doi:10 1007/978-1-4613-1733-3_1

Straif K, Loomis D, Guyton K, Grosse Y, Lauby-Secretan B, El Ghissassi F Bouvard V, Benbrahim-Tallaa L, Guha N, Mattock H. 2014. Future priorities for the IARC Monographs. Lancet Oncol 15: 683-684. doi:10.1016/ S1470-2045(14)70168-8

Sumner SC, Fennell TR, Moore TA, Chanas B, Gonzalez F, Ghanayem BI. 1999. Role of cytochrome P450 2E1 in the metabolism of acrylamide and acrylonitrile in mice. Chem Res Toxicol 12: 1110-1116. doi:10 $.1021 / \mathrm{tx} 990040 \mathrm{k}$

\section{Genome Research}

www.genome.org 
Takatsuki S, Nemoto S, Sasaki K, Maitani T. 2003. Determination of acrylamide in processed foods by LC/MS using column switching. Shokuhin Eiseigaku Zasshi 44: 89-95. doi:10.3358/shokueishi.44.89

Tareke E, Rydberg P, Karlsson P, Eriksson S, Törnqvist M. 2002. Analysis of acrylamide, a carcinogen formed in heated foodstuffs. I Agric Food Chem 50: 4998-5006. doi:10.1021/jf020302f

Todaro GJ, Green H. 1963. Quantitative studies of the growth of mouse embryo cells in culture and their development into established lines. J Cell Biol 17: 299-313. doi:10.1083/jcb.17.2.299

Tomkova M, Tomek J, Kriaucionis S, Schuster-Böckler B. 2018. Mutational signature distribution varies with DNA replication timing and strand asymmetry. Genome Biol 19: 129. doi:10.1186/s13059-018-1509-y

Virk-Baker MK, Nagy TR, Barnes S, Groopman J. 2014. Dietary acrylamide and human cancer: a systematic review of literature. Nutr Cancer 66: 774-790. doi:10.1080/01635581.2014.916323

Von Tungeln LS, Churchwell MI, Doerge DR, Shaddock JG, McGarrity LJ, Heflich RH, da Costa GG, Marques MM, Beland FA. 2009. DNA adduct formation and induction of micronuclei and mutations in $\mathrm{B}_{6} \mathrm{C}_{3} \mathrm{~F}_{1} / \mathrm{Tk}$ mice treated neonatally with acrylamide or glycidamide. Int $J$ Cancer 124: 2006-2015. doi:10.1002/ijc. 24165

Von Tungeln LS, Doerge DR, da Costa GG, Marques M M, Witt WM, Koturbash I, Pogribny IP, Beland FA. 2012. Tumorigenicity of acrylamide and its metabolite glycidamide in the neonatal mouse bioassay. Int J Cancer 131: 2008-2015. doi:10.1002/ijc.27493
Westcott PMK, Halliwill KD, To MD, Rashid M, Rust AG, Keane TM, Delrosario R, Jen K-Y, Gurley KE, Kemp CJ, et al. 2014. The mutational landscapes of genetic and chemical models of Kras-driven lung cancer. Nature 517: 489-492. doi:10.1038/nature13898

Wilson KM, Bälter K, Adami HO, Grönberg H, Vikström AC, Paulsson B, Törnqvist M, Mucci LA. 2009. Acrylamide exposure measured by food frequency questionnaire and hemoglobin adduct levels and prostate cancer risk in the Cancer of the Prostate in Sweden Study. Int J Cancer 124: $2384-2390$. doi: $10.1002 / \mathrm{ijc} .24175$

Xie J, Terry KL, Poole EM, Wilson KM, Rosner BA, Willett WC, Vesper HW, Tworoger SS. 2013. Acrylamide hemoglobin adduct levels and ovarian cancer risk: a nested case-control study. Cancer Epidemiol Biomarkers Prev 22: 653-660. doi:10.1158/1055-9965.EPI-12-1387

Zhang W, He H, Zang M, Wu Q, Zhao H, Lu LL, Ma P, Zheng H, Wang N, Zhang Y, et al. 2017. Genetic features of aflatoxin-associated hepatocellular carcinoma. Gastroenterology 153: 249-262.e2. doi:10.1053/j.gastro 2017.03.024

Zhivagui M, Korenjak M, Zavadil J. 2017. Modelling mutation spectra of human carcinogens using experimental systems. Basic Clin Pharmacol Toxicol 121: 16-22. doi:10.1111/bcpt.12690

Received July 31, 2018; accepted in revised form February 1, 2019. 


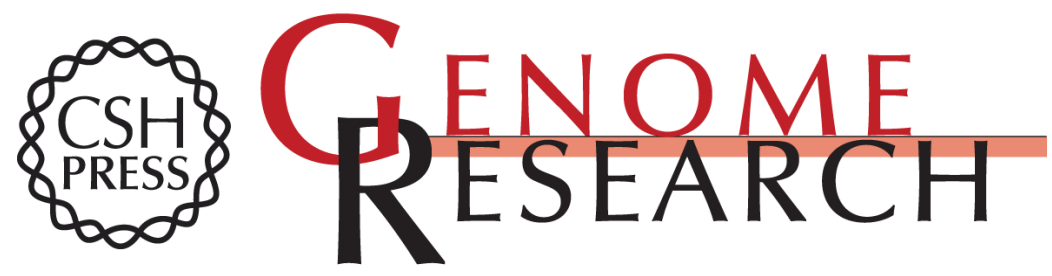

\section{Experimental and pan-cancer genome analyses reveal widespread contribution of acrylamide exposure to carcinogenesis in humans}

Maria Zhivagui, Alvin W.T. Ng, Maude Ardin, et al.

Genome Res. 2019 29: 521-531 originally published online March 7, 2019

Access the most recent version at doi:10.1101/gr.242453.118

Supplemental Material

References

Open Access

Creative Commons

License

Email Alerting Service
http://genome.cshlp.org/content/suppl/2019/03/05/gr.242453.118.DC1

This article cites 62 articles, 17 of which can be accessed free at: http://genome.cshlp.org/content/29/4/521.full.html\#ref-list-1

Freely available online through the Genome Research Open Access option.

This article, published in Genome Research, is available under a Creative Commons License (Attribution-NonCommercial 4.0 International), as described at http://creativecommons.org/licenses/by-nc/4.0/.

Receive free email alerts when new articles cite this article - sign up in the box at the top right corner of the article or click here.

\section{Affordable, Accurate Sequencing.}

